\title{
Evaluación de impacto ambiental de centro de transformación y gestión de residuos sólidos agrícolas en la provincia de Almería (España)
}

\author{
Environmental impact of an agricultural solid waste disposal \\ and transformation plant in the Province of Almería (Spain)
}

Informes de la Construcción Vol. 62, 518, 79-93, abril-junio 2010 ISSN: 0020-0883 elSSN: 1988-3234 doi: 10.3989/ic.08.028

\author{
$\underline{\text { A. J. Callejón }}^{(*)}$, A. Carreño ${ }^{(*)}$, J. Sánchez-Hermosilla ${ }^{(*)}$, J. Pérez ${ }^{(*)}$
}

\section{RESUMEN}

En la agricultura intensiva almeriense, el tipo de residuo que se genera es muy variado y las características del mismo hacen que no pueda ser aprovechado de modo directo por los animales ya que la composición mixta en muchos casos (materia vegetal y rafias) o el tipo de algunos residuos, hace que se vaya pensando en una gestión discriminada del residuo para obtener de cada tipo un máximo de aprovechamiento y una minimización en el impacto que causen, pasando de un concepto de residuo al de subproducto susceptible de diferentes aplicaciones como medio de producción. Para el tratamiento, transformación y gestión de los residuos sólidos agrícolas (biomasa, materiales plásticos, envases de fertilizantes y fitosanitarios, maderas y alambres, aceites de desecho y sustratos) los recursos naturales a emplear serán agua y mano de obra, principalmente. Todo ello, para obtener compost vegetal, granza a partir de materiales plásticos, materiales metálicos clasificados, maderas y otros materiales de similar composición clasificados, envases de productos fitosanitarios y fertilizantes clasificados y gestión de aceites de desecho. Los efluentes que se emitirán serán ruidos, levantamiento de polvo, emisión de olores, aguas residuales de frutas, aguas procedentes del lavado de camiones y contenedores, aguas de lavado de envases de productos fitosanitarios, aguas procedentes de la humectación del material vegetal. Mediante la evaluación de impacto ambiental se concluye que es mejor reciclar y compostar que obtener energía a partir de los residuos, además se propone un sistema de impermeabilización con hormigón polímero para controlar los efluentes de productos fitosanitarios.

\section{3-95}

Palabras clave: compost, hormigón polímero, impacto ambiental, invernaderos, residuos.
SUMMARY

The wastes generated by the intensive agriculture systems of the Province of Almería (Spain) are very varied, and they frequently cannot be directly reused; for example, mixtures of plant material and plastic raffia cannot be used as animal feed. A less indiscriminate form of management of these wastes, however, could lead to their being turned into usable products. This would also reduce their environmental impact. The natural resources needed for the treatment, transformation and management of solid agricultural wastes such as biomass, plastics, fertilizer and crop protection product containers, wood, wire, waste oils and waste substrates, are largely water and manpower. The final products that might be produced are plant compost, plastic granules, classified metal metallic materials, separated fertilizer and crop protection product containers, and waste oils that could be further treated. The problems associated with such waste management would be noise, the production of dust and smells, the leaching of liquids from waste plants and fruit, the dirty water left over after washing out trucks and fertilizer and crop protection product containers etc., and the waste water produced by the wetting of plant material. The assessment of the environmental impact of a potential waste treatment plant shows that it would be better to recycle and compost wastes than to try to obtain energy from them through combustion. A polymer concrete system for the control of effluents produced from crop protection products is also discussed.

Keywords: compost, polymer concrete, environmental impact, greenhouses, wastes. 


\section{INTRODUCCIÓN}

El modelo de agricultura almeriense es intensivo tanto en producciones como "inputs", todo ello bajo condiciones deseables de respeto del medio que nos rodea. La magnitud de los residuos es grande tanto en hetereogeneidad como en cantidad (Figura 1), ya que son 27.000 ha (Sanjuán, 2004) (1) de cultivo bajo plástico las existentes en la provincia de Almería.

En 1995, Hall y House (2) veían en la biomasa un combustible ambientalmente aceptable para el futuro. Dicha biomasa se encuentra en forma tradicional de leña, residuos vegetales y estiércol (Velázquez-Martí, 2006) (3). Si estos son mal utilizados pueden ser perjudiciales para el medio ambiente, por el contrario si se utilizan de forma eficiente y sostenible, la energía de la biomasa tiene numerosos beneficios ambientales y sociales en comparación con los combustibles fósiles. Los efectos beneficiosos incluyen el control de residuos, el reciclaje de nutrientes, la creación de empleo, la utilización de los excedentes de tierras agrícolas en los países industrializados, suministro eléctrico de zonas rurales, mejora de la gestión de tierras y reducción de contaminación de $\mathrm{CO}_{2}$ (Velázquez-Martí y Fernández-González, 2009) (4).

Para Camacho et al. (2000) (5) lo que se pretendía con la implantación del plan de higiene rural de la Comarca Agraria de El Campo de Níjar en Almería es que todos los residuos generados por los invernaderos fuesen recogidos, reciclados y reutilizados, hecho éste, que en la actualidad poco a poco se está consolidando en dicha comarca. De hecho, la solidez ecológica puede traer crecimiento económico, pero necesita del aprendizaje de la sociedad y estructuras institucionales, para así ser eficaz (Lightfoot y Noble, 2001; Velázquez-Martí y Annevelink, 2009; Velázquez-Martí y FernándezGonzález, 2010) (6-8).

López y Rodríguez (2003) (9) mediante el prototipo de una planta integral para reciclado de envases fitosanitarios garantizaron la destoxificación de los mismos en el modelo de horticultura intensivo de Almería; para ello recogían todas las aguas de lavado de

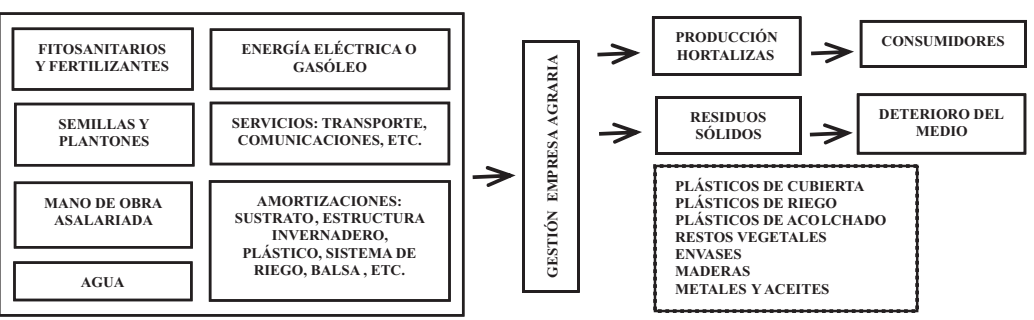

envases y las trataban químicamente, además de con ozono.

Matarán (2006) (10), describe que en la zona de la Costa de Granada, sólo se están abordando tres grupos de problemas en la agricultura bajo plástico, el primero la gestión de los envases, el segundo la extracción de arena y el tercero la salinización de los acuíferos.

Más recientemente, Antón et al. (2007) (11), evaluaron el impacto ambiental relacionado con la horticultura protegida de Cataluña y lo compararon con otras actividades cercanas. Los resultados obtenidos demostraron que la horticultura intensiva se encuentra más o menos igual que las actividades de zonas urbanas, desde el punto de vista medioambiental.

En la agricultura bajo plástico, el uso excesivo de fertilizantes minerales afecta a la calidad del suelo, dando lugar a problemas ambientales, por el contrario la enmienda orgánica puede mejorar la calidad del suelo y al mismo tiempo proporcionar nutrientes a los cultivos, pudiendo ser los purines de cerdo y los compost de origen vegetal una alternativa (Ros et al., 2007) (12).

No sólo se debe entender, que la biomasa de origen vegetal procedente de los invernaderos sirve para hacer compost, de hecho Elias (2007) (13) considera y analiza las diferentes posibilidades de la biomasa como recurso energético, pero sin olvidar que aunque el término biomasa incluye la materia orgánica producida como resultado de la fotosíntesis, además de residuos de origen orgánico de municipios e industrias y que los sistemas de combustión de biomasa son poco contaminantes y ofrecen una protección significativa del medio ambiente por la reducción de la contaminación de gases de efecto invernadero, sí contaminan,en cambio, sus cenizas y otros gases emitidos (Demirbas, 2005; Velázquez-Martí et al 2010) (14) (15).

En cuanto a las metodologías utilizadas en la evaluación, Gómez (1988) (16) desarrolló, para posteriormente mejorar en el año 1999 (17), un método de evaluación de impacto ambiental para cualquier actividad, agraria o no, basado en matrices de interacción, cruzando las acciones humanas con los factores que componen el entorno, tales como aire, suelo y agua, entre otros. Igualmente, Girardin et al. (2000) (18) desarrollaron el método AGRO-ECO para evaluación de impactos agrícolas utilizando similares matrices.

Braband et al. (2003) (19), analizaron 7 métodos de evaluación de impacto ambiental en agricultura, tales como: Ecopoints coger Austria, Criteria for environmentally sound 
agricultura, At the Danish Institute for Agricultura a method, The French approach Solagro, The method Developer by Frieben, The Nature Balance Scheme y The biodiversity Yardstick Developer at the Centre for agricultura and Envioronment in Utrech; Ilegando a la conclusión de que todos éstos quieren contribuir al desarrollo de la agricultura ecológica y alertando de la posibilidad de seguir desarrollando y mejorando instrumentos eficientes para evaluar el impacto ambiental de las actividades agrícolas.

Krishnan et al. (2004) (20) evaluaron el impacto ambiental mediante un método que lo cuantifica teniendo en cuenta factores multivariables e interaccionando valores económicos, ambientales y la salud de los ciudadanos.

También, Payraudeau y Van Der Werf (2005) (21) y Van Der Werf et al. (2007) (22), aplicaron y compararon 6 métodos en agricultura: Environmental risk mapping (ERM), Life cycle analysis (LCA), Environmental impact assessment (EIA), Multi-agent system (MAS), Multiple linear programming (LP) approaches y Agro-environmental indicators (AEI); concluyendo que los métodos deben ser utilizados con gran cautela, debiéndose considerar primeramente qué método es el más adecuado según las necesidades de la acción humana a evaluar.

A su vez, para la evaluación de impacto ambiental de las zonas rurales en Francia Galan et al. (2007) (23) aplicaron y compararon 5 métodos denominados "General characteristics of the IDEA ('Indicateur de Durabilite' des Exploitations Agricoles"), DIAGE (Diagnostic Global d'Exploitation), DIALECTE (Diagnostic Liant Environnement et CTE), DIALOGUE (Diagnostic agri-environnemental global d'exploitation) e INDIGO ("indicateurs de diagnostic global a ' la parcelle)", todos ellos utilizando matrices de interacción.

Independientemente de los métodos anteriores, Anderson y Thompson (2007) (24) diseñaron un método de control multivariable que identificaba los impactos en las zonas rurales de forma muy rápida para poder así dar respuestas urgentes a las autoridades y/o sociedad.

A modo de método, Willians et al. (2008) (25) clasifican las zonas rurales en agroecozonas, proporcionando un marco de conocimiento para los posibles usos de zonas rurales en USA, mediante fotografía satélite de parámetros ambientales y agrícolas. Algo, más sencillo, sin tener en cuenta valores ecológicos, se hizo en España a mediados de los años 80 mediante el Átlas Agroclimático Nacional (De León Llamazares, 1986) (26), el cual enunciaba los posibles usos agrícolas de todas las zonas rurales de España mediante métodos analíticos.

En general, todos los autores citados anteriormente coinciden en que los métodos deben ser adaptados en función del entorno donde se aplican y las acciones que se realicen.

Así pués, para la gestión integral de los residuos sólidos agrícolas generados "por los invernaderos" (Figura 1) han sido varias las plantas de tratamiento realizadas en la provincia de Almería, la mayoría de ellas con el fin de obtener energía a partir de los restos vegetales (biogás), además no se ha acabado de dar una solución definitiva al acopio de envases fitosanitarios y sí al reciclado de plástico que funciona muy bien. Dicha actividad está sujeta por Ley a evaluación de impacto ambiental (27) (28).

El objetivo principal de este trabajo es conseguir plenamente un desarrollo agrícola sostenible y respetuoso con el medio ambiente mediante el estudio de impacto ambiental tanto en la fase de estudios previos, construcción y explotación de la actividad de un centro de transformación y gestión de residuos, todo ello para mejorar la calidad de vida de los ciudadanos (Figura 2).

\section{MATERIAL Y MÉTODOS}

La zona en estudio queda localizada en el Sureste de España (Figura 3).

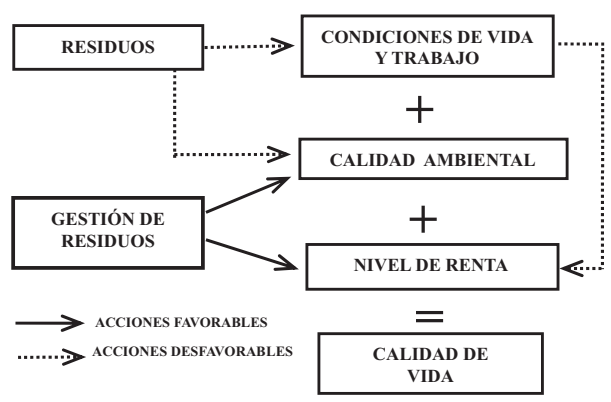

2

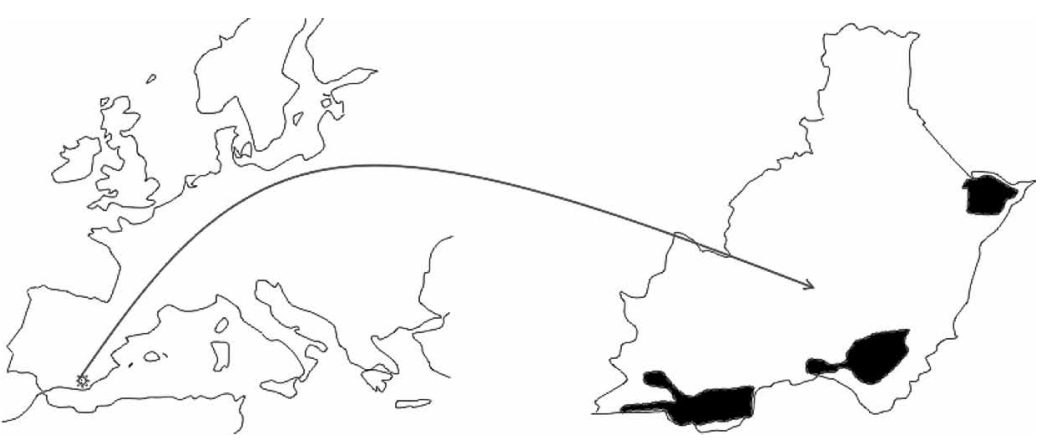
duos.

3. Localización del $80 \%$ de invernaderos (Callejón-Ferre et al., 2009a) (29).
2. Ventajas de la gestión de resi- 
La superficie actual de invernaderos se aproxima a las 30.000 ha y la tipología de los mismos (generadores de residuos) corresponde principalmente al invernadero "Tipo Almería plano y Tipo Almería raspa y amagado" (Figura 4) en un 96,5\% (Fernández y Pérez) (30), con una producción final agraria estabilizada alrededor de los 1.500 millones de euros (Cajamar) (31).

De entre los tres grandes grupos de residuos que se pueden generar en la provincia, los vegetales ascienden a 28,5 th ha ${ }^{-1} \cdot a n ̃ o{ }^{-1}$, como media, que corresponderían con $114 \mathrm{~m}^{3} \cdot \mathrm{ha}^{-1} \cdot \mathrm{anno}^{-1}$ (Manzano, 2007) [33]. Multiplicando por las 27.000 ha invernadas (Sanjuán, 2004) (1) se obtienen 769.500 t·año-1 (Callejón-Ferre y LópezMartínez, 2009) (34) que en volumen suponen $3.078 .000 \mathrm{~m}^{3} \cdot \mathrm{anno}^{-1}$ de residuo vegetal.

Igualmente, la cantidad de envases fitosanitarios que son capaces de generarse en una hectárea de invernadero es de 93,1 envases $\cdot \mathrm{ha}^{-1} \cdot \mathrm{añno}^{-1}$, teniendo en cuenta envases de $1,6 \mathrm{~kg}$ o I (Camacho et al., 2000) (5). De éstos 68,1 envases son de plástico y el resto de otros materiales. En total se producen 2.513.700 envases·año ${ }^{-1}$.

Finalmente, en cuanto al plástico de cubierta, aproximadamente se consumen $2 \mathrm{t} \cdot \mathrm{ha}^{-1}$, renovándose cada 3 años (Camacho et al., 2000) (5), por lo tanto, si suponemos que la reposición va a ser escalonada de forma lineal, el plástico para reciclar será de 18.000 t $\cdot a n ̃ o^{-1}$.

Con la cantidad de residuos que es capaz de generar este sector y siguiendo los criterios de Payraudeau y Van Der Werf (2005) (21) y Van Der Werf et al. (2007) (22) la metodología elegida para este trabajo ha sido la desarrollada por Gómez (1999) (17), teniendo en cuenta la Ley estatal (27) y autonómica (28) de evaluación de impacto ambiental.

\section{RESULTADOS Y DISCUSIÓN}

El método de Gómez (1999) (17), se basa en matrices de interacción al igual que los métodos definidos por Girardin et al. (2000) (18), Braband et al. (2003) (19), Krishnan et al. (2004) (20), Payraudeau y Van Der Werf (2005) (21) y Van Der Werf et al. (2007) (22), Galán et al. (2007) (23) y Anderson y Thompson (2007) (24).

\subsection{Matriz de impactos}

Las acciones irán encaminadas a la construcción de un centro de transformación y gestión de residuos sólidos agrícolas. La transformación, consistirá fundamentalmente, en el compostaje de los residuos vegetales.

Del estudio previo de las acciones, el inventario ambiental y los factores ambientales se ha obtenido la identificación preliminar de posibles impactos (Figura 5), seguidamente la matriz depurada (Figura 6) para finalmente conseguir la matriz y caracterización de los impactos más significativos (Figura 7).

En la Figura 7 destacan como impactos más significativos el emplazamiento del centro de transformación sobre la población en la fase de estudios previos; el movimiento de tierra sobre la erosión en la fase de construcción; la recogida y transporte de residuos sólidos agrícolas sobre la dinámica de cauces, medio marino y costero, sobre la población y áreas de mercado en las fases de explotación; generación de polvo en el transporte sobre la deposición (cubiertas de invernaderos principalmente) en la fase de explotación; aguas de los envases fitosanitarios sobre la edafología del entorno en la fase de explotación; y el efecto de la mano de obra contratada sobre la población en la fase de explotación.

Para poder caracterizar los impactos y conocer su forma de actuar se hace necesario valorar sus atributos (Tabla 1, pág. 85) que posteriormente serán utilizados para el cálculo de la incidencia estandarizada en el punto 3.5.

\subsection{Indicadores de impacto}

El problema o beneficio que se causa al interaccionar acciones con factores ambientales es analizado mediante los indicadores y sus unidades de medida (Tabla 2, pág. 85).

\subsection{Estimación de la magnitud de los impactos}

Seguidamente habría que diferenciar entre impactos cuantificables en unidades heterogéneas (Tabla 3, pág. 86) en y los cuantificables en unidades homogéneas o de origen cualitativo (Tabla 4, pág. 86). laterales

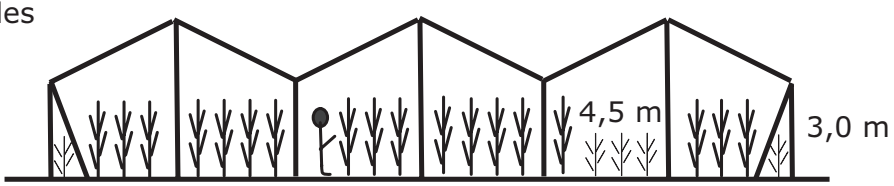




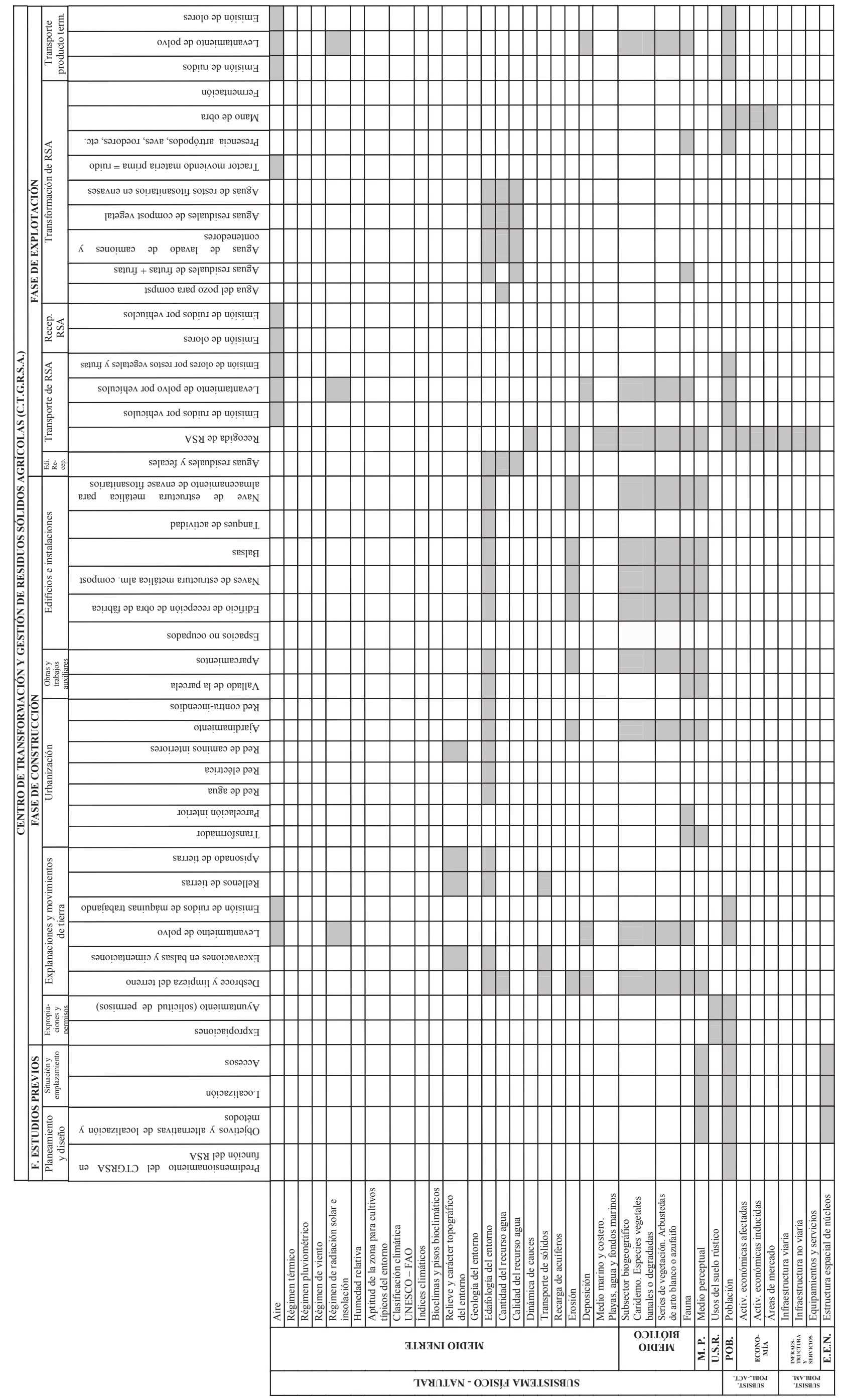




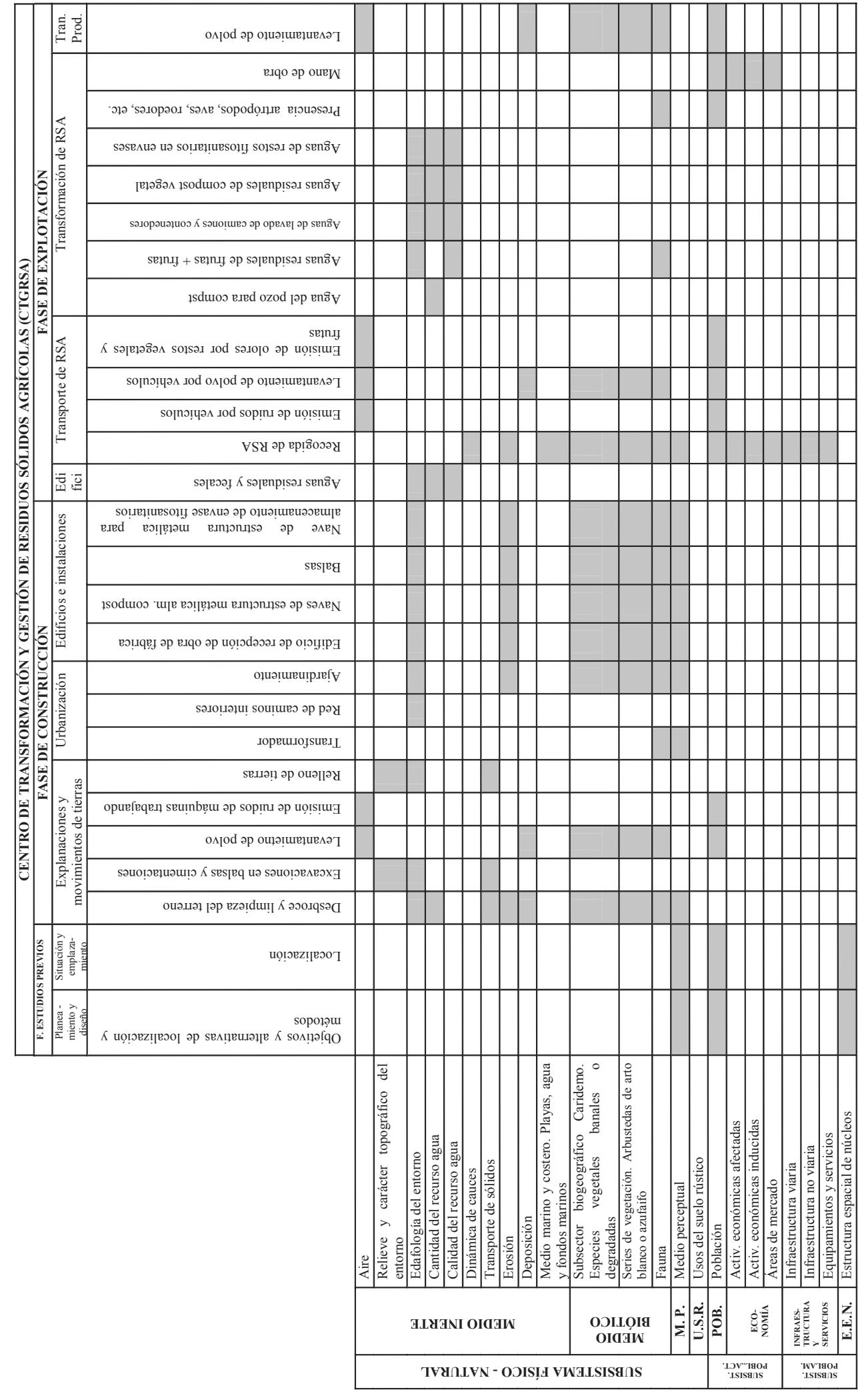




\subsection{Búsqueda y construcción de funciones de transformación. Valoración de impactos en unidades homogéneas}

Aquellos impactos medibles en unidades heterogéneas necesitan de funciones de transformación para poder pasar a ser medibles en unidades homogéneas, para lo cual se utilizan las funciones de la Figura 8, pág. 87, obtenidas de Gómez (1999) (17). Una vez transformados los impactos se agrupan todos en unidades homogéneas en la Tabla 5, pág. 86, con breves comentarios explicativos en cada uno de ellos.

\subsection{Incidencia estandarizada de impactos}

Teniendo en cuenta la caracterización de los impactos a través de una serie de atributos (Tabla 1), se calculará la incidencia mediante la suma ponderada de los valores de los atributos para cada impacto, afectados a su vez por un coeficiente según la importancia de cada atributo. Posteriormente utilizando la fórmula [1] se obtendrá el valor de la incidencia estandarizada.

Actuando de igual modo que con el ejemplo del impacto de la localización sobre la población (Tabla 6), se obtendrán los resultados del resto de impactos (Tabla 7, pág. 87).

Incid $_{\text {estandarizada }}=\frac{{\text { Incid }- \text { Incid }_{\text {min }}}_{\text {Incid }_{\text {máx }}}}{}$
7. Matriz de impactos más significativos. Matriz depurada II.

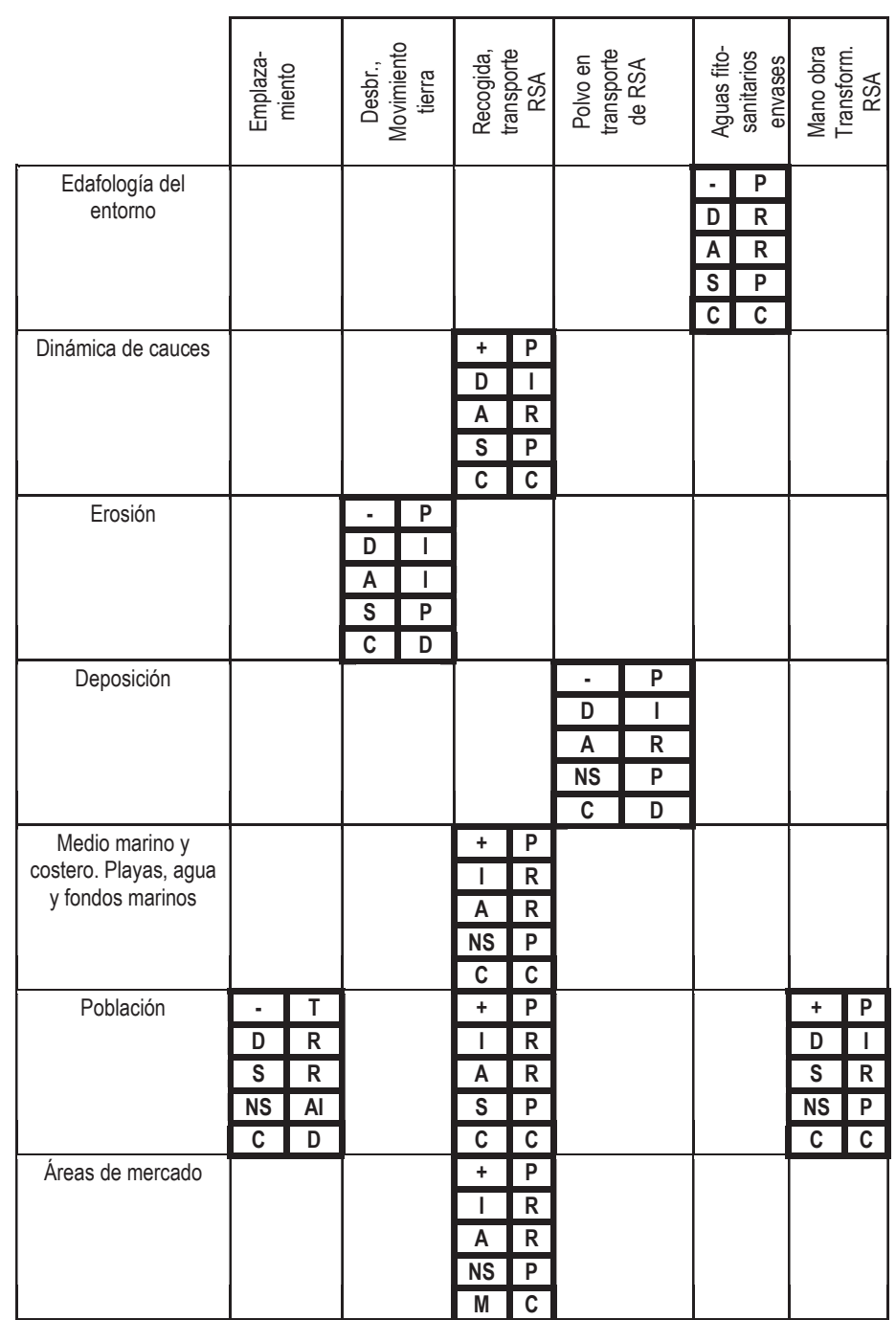

Tabla 1

Caracterización de impactos y valoración de sus atributos

\begin{tabular}{|c|c|c|c|c|c|}
\hline Caracterización & Atributo & Valoración $\times f$ & Caracterización & Atributo & Valoración $\times f$ \\
\hline Signo & Positivo (+) / Negativo (-) & {$[+=1$ y $-=3] \times 1$} & Persistencia & Temporal (T) / Permanente (P) & {$[\mathrm{T}=1$ y $\mathrm{P}=3] \times 1$} \\
\hline Inmediatez & Directo (D) / Indirecto (I) & {$[D=3$ e $I=1] \times 3$} & Reversibilidad & Reversible (R) / Irreversible (I) & {$[\mathrm{R}=1$ e $\mathrm{I}=3] \times 3$} \\
\hline Acumulación & Simple (S) / Acumulativo (A) & {$[\mathrm{A}=3$ y $\mathrm{S}=1] \times 2$} & Recuperabilidad & Recuperable (R) / Irrecuperable (I) & {$[\mathrm{R}=1$ e $\mathrm{I}=3] \times 1$} \\
\hline Sinergia & Sinérgico (S) / No sinérgico (NS) & {$[\mathrm{S}=3$ y NS=1]x1 } & Periodicidad & Periódico (P) / Aparición irregular (Al) & {$[\mathrm{P}=3$ y $\mathrm{Al}=1] \times 1$} \\
\hline Momento & $\begin{array}{c}\text { Corto plazo (C) / Medio plazo (M) } \\
\text { / Largo plazo (L) }\end{array}$ & {$[\mathrm{C}=3, \mathrm{M}=2$ y $\mathrm{L}=1] \times 1$} & Continuidad & Continuo (C) / Discontinuo (D) & {$[C=3$ y $D=1] \times 1$} \\
\hline
\end{tabular}

Tabla 2

Indicadores de impacto

\begin{tabular}{|c|c|c|c|}
\hline \multicolumn{2}{|c|}{ IMPACTO } & \multirow{2}{*}{ Indicador } & \multirow{2}{*}{$\begin{array}{l}\text { Unidad } \\
\text { de medida }\end{array}$} \\
\hline Acción & Factor & & \\
\hline Localización & Población & Aceptación social del proyecto & $\%$ \\
\hline $\begin{array}{l}\text { Desbroce y } \\
\text { limpieza }\end{array}$ & Erosión & Desplazamiento de materiales arrastrados por agua o aire & t/ha·año \\
\hline Recogida de RSA & $\begin{array}{l}\text { Dinámica } \\
\text { cauces }\end{array}$ & \% en volumen de ocupación de cauces por RSA & $\%$ \\
\hline $\begin{array}{l}\text { Levantamiento } \\
\text { polvo }\end{array}$ & Deposición & $\begin{array}{c}\text { Variación de volumen de deposición, en zonas sensibles, con respecto a las condiciones } \\
\text { naturales }\end{array}$ & $\%$ \\
\hline Aguas fitosanitarios & Edafología & $\propto g$ fitosanitario / kg de suelo & $\propto \mathrm{cg} / \mathrm{kg}$ \\
\hline
\end{tabular}


Tabla 3

Impactos valorables cuantitativamente (en unidades heterogéneas)

\begin{tabular}{|l|c|c|}
\hline \multicolumn{1}{|c|}{ IMPACTO } & $\begin{array}{c}\text { Valor sin } \\
\text { proyecto }\end{array}$ & $\begin{array}{c}\text { Valor con } \\
\text { proyecto }\end{array}$ \\
\hline De localización sobre la población & $0 \%$ & $7,5 \%$ \\
\hline Del desbroce y limpieza sobre la erosión & $0,6 \mathrm{~kg} / \mathrm{m}^{2} \cdot$ año & $1,45 \mathrm{~kg} / \mathrm{m}^{2} \cdot$ año \\
\hline De la recogida de RSA sobre la dinámica de cauces & $22 \%$ & $0 \%$ \\
\hline Del levantamiento de polvo sobre la deposición & $0 \%$ & $62 \%$ \\
\hline De las aguas de los envases de fitosanitarios sobre la edafología & $0 \propto \mathrm{g} / \mathrm{kg}$ & $2,4 \propto \mathrm{gg} / \mathrm{kg}$ \\
\hline De la mano de obra sobre la población & $85 \%$ & $90 \%$ \\
\hline
\end{tabular}

Tabla 4

Impactos valorables cualitativamente (directamente en unidades homogéneas)

\begin{tabular}{|l|c|c|}
\hline \multicolumn{1}{|c|}{ IMPACTO } & Valor sin proyecto & Valor con proyecto \\
\hline De la recogida de RSA sobre el medio marino y costero & 0,44 & 0,98 \\
\hline De la recogida de RSA sobre la población & 0,3 & 0,96 \\
\hline De la recogida de RSA sobre las áreas de mercado & 0,5 & 0,94 \\
\hline
\end{tabular}

Tabla 5

Valoración de la magnitud de los impactos en unidades homogéneas

\begin{tabular}{|c|c|c|c|c|}
\hline IMPACTO & $\begin{array}{c}\text { Sin } \\
\text { proyecto }\end{array}$ & $\begin{array}{c}\text { Con } \\
\text { proyecto }\end{array}$ & $\begin{array}{l}\text { Magnitud } \\
\text { del impacto }\end{array}$ & Comentario \\
\hline $\begin{array}{l}\text { De localización } \\
\text { sobre la población }\end{array}$ & 1,00 & 0,79 & $-0,21$ & $\begin{array}{l}\text { Se supone que el \% de población afectado } \\
\text { negativamente es de una pedanía cercana con una muy } \\
\text { baja densidad de población ( } 250 \text { habitantes })\end{array}$ \\
\hline $\begin{array}{l}\text { Del desbroce y limpieza } \\
\text { sobre la erosión }\end{array}$ & 0,97 & 0,74 & $-0,23$ & $\begin{array}{c}\text { Se producirá sólo en la ubicación del Centro de Gestión } \\
\text { de Residuos Sólidos Agrícolas }\end{array}$ \\
\hline $\begin{array}{l}\text { De la recogida de RSA } \\
\text { sobre la dinámica de } \\
\text { cauces }\end{array}$ & 0,02 & 0,98 & 0,96 & $\begin{array}{c}\text { El control y limpieza es fundamental, de lo contrario se } \\
\text { podría contaminar la única reserva marina de Andalucía, } \\
\text { ubicada en el Parque Natural }\end{array}$ \\
\hline $\begin{array}{l}\text { Del levantamiento } \\
\text { de polvo sobre la } \\
\text { deposición }\end{array}$ & 1,00 & 0,62 & $-0,38$ & $\begin{array}{c}\text { Afectaría sobre todo en la fase de construcción, } \\
\text { posteriormente no }\end{array}$ \\
\hline $\begin{array}{l}\text { De las aguas de los } \\
\text { envases de fitosanitarios } \\
\text { sobre la edafología }\end{array}$ & 1,00 & 0,66 & $-0,34$ & $\begin{array}{l}\text { El control de residuos evitará la contaminación del suelo } \\
\text { y posteriormente los acuíferos }\end{array}$ \\
\hline $\begin{array}{l}\text { De la mano de obra } \\
\text { sobre la población }\end{array}$ & 0,97 & 1,00 & 0,03 & Generación de empleo \\
\hline $\begin{array}{l}\text { De la recogida de RSA } \\
\text { sobre el medio marino } \\
\text { y costero }\end{array}$ & 0,44 & 0,98 & 0,54 & $\begin{array}{c}\text { El control y limpieza es fundamental, de lo contrario se } \\
\text { podría contaminar la única reserva marina de Andalucía, } \\
\text { ubicada en el Parque Natural }\end{array}$ \\
\hline $\begin{array}{l}\text { De la recogida de RSA } \\
\text { sobre la población }\end{array}$ & 0,30 & 0,96 & 0,66 & $\begin{array}{c}\text { El control de residuos provocará mejor calidad de vida, } \\
\text { es decir, salubridad }\end{array}$ \\
\hline $\begin{array}{l}\text { De la recogida de RSA } \\
\text { sobre las áreas de } \\
\text { mercado }\end{array}$ & 0,50 & 0,94 & 0,44 & $\begin{array}{l}\text { El control de residuos es posible que repercuta en un } \\
\text { aumento del precio del producto elaborado en las } \\
\text { explotaciones hortícolas }\end{array}$ \\
\hline
\end{tabular}

Tabla 6

Ejemplo de cálculo de la incidencia del impacto "De localización sobre la población"

\begin{tabular}{|c|c|c|c|c|c|c|c|}
\hline $\begin{array}{c}\text { Impac- } \\
\text { to }\end{array}$ & \multicolumn{2}{c|}{ Incidencia } & \multicolumn{2}{c|}{$\begin{array}{c}\text { Incidencia } \\
\text { mínima }\end{array}$} & \multicolumn{2}{c|}{$\begin{array}{c}\text { Incidencia } \\
\text { máxima }\end{array}$} \\
\hline- & T & $3 \times 1$ & 1 & $1 \times 1$ & $1 \times 1$ & $3 \times 1$ & $3 \times 1$ \\
\hline D & R & $3 \times 3$ & $1 \times 3$ & $1 \times 3$ & $1 \times 3$ & $3 \times 3$ & $3 \times 3$ \\
\hline S & R & $1 \times 2$ & 1 & $1 \times 2$ & $1 \times 1$ & $3 \times 2$ & $3 \times 1$ \\
\hline $\begin{array}{c}\text { N } \\
\text { S }\end{array}$ & I & 1 & 1 & $1 \times 1$ & $1 \times 1$ & $3 \times 3$ & $3 \times 1$ \\
\hline C & D & 3 & 1 & $1 \times 1$ & $1 \times 1$ & $3 \times 3$ & $3 \times 1$ \\
\hline $\begin{array}{c}\text { Sub- } \\
\text { total }\end{array}$ & 18 & 7 & 8 & 7 & 24 & 21 \\
\hline \multicolumn{2}{|c|}{ Total } & \multicolumn{2}{|c|}{25} & \multicolumn{2}{|c|}{15} & \multicolumn{3}{|c|}{45} \\
\hline
\end{tabular}

\subsection{Valor final y enjuiciamiento de los impactos}

Con el valor de la incidencia estandarizada (Tabla 7) multiplicado por el de la magnitud (Tabla 5) se obtiene la calificación del impacto (Tabla 8, pág. 88).

\subsection{Totalización del impacto del proyecto}

Para calcular el valor ponderado del impacto hay que darle el peso relativo que tendría cada uno de ellos (Tabla 9, pág. 88), de esta manera se corrigen los posibles desequilibrios entre valores.

Finalmente el impacto final del proyecto sería de 0,140 y, por lo tanto, sería viable desde el punto de vista ambiental, teniendo en cuenta las medidas correctoras y de seguimiento - mantenimiento que habría que desarrollar.

Evidentemente, este valor positivo indica que se mejora la calidad de vida de los ciudadanos resólviendose un problema ambiental, coincidiendo con lo postulado en 1995 por Hall y House (2) además de la mejora económica como consecuencia de una acción ecológica (Lightfoot y Noble (2001) (6). 
Calidad
Ambiental

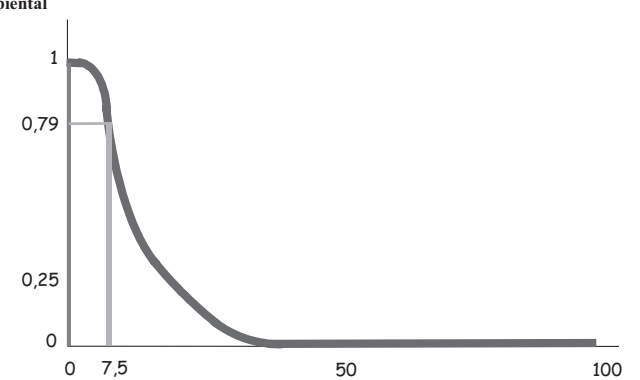

Calidad

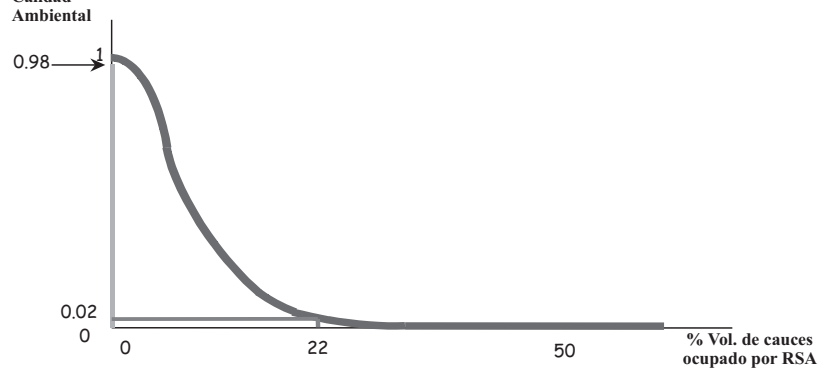

Calidad
Ambiental

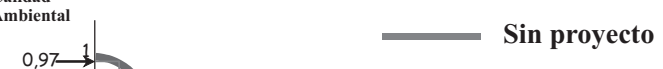

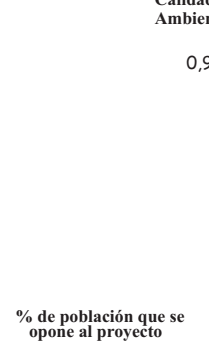

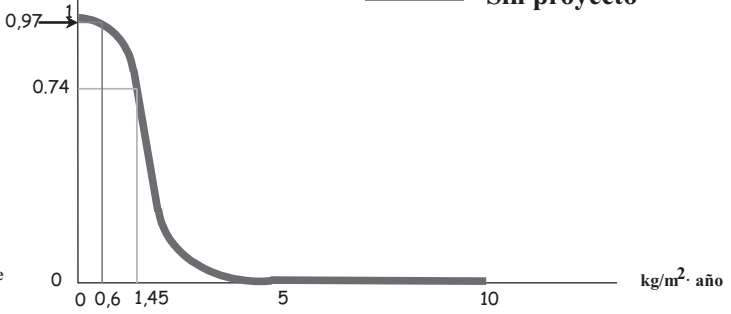

Calidad

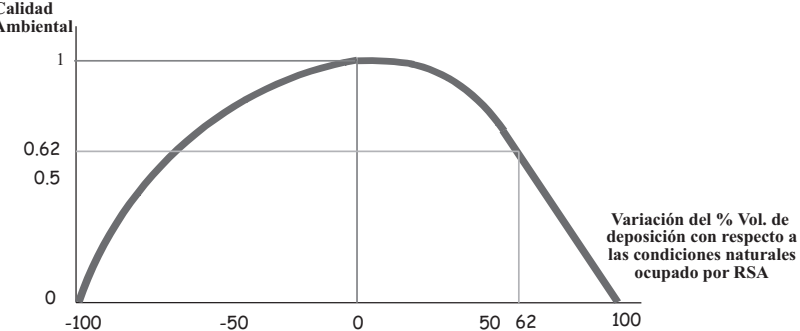

Calidad
Ambiental
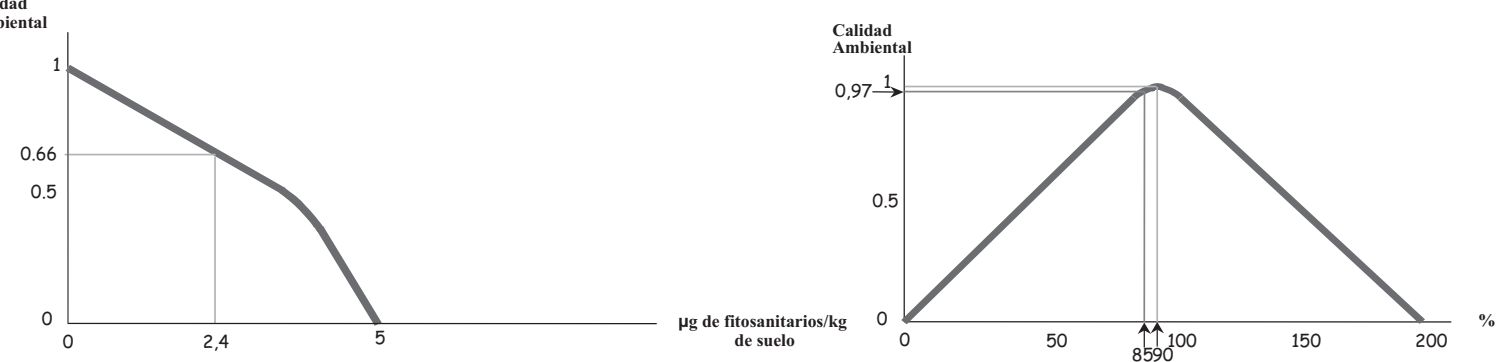

Tabla 7

Resultados de la incidencia estandarizada de los impactos

\begin{tabular}{|c|c|c|c|}
\hline IMPACTO & $\begin{array}{l}\text { Valor de } \\
\text { la } \\
\text { incidencia }\end{array}$ & $\begin{array}{l}\text { Valor } \\
\text { Estandarizado } \\
\text { de la incidencia }\end{array}$ & COMENTARIO \\
\hline $\begin{array}{l}\text { De localización sobre } \\
\text { la población }\end{array}$ & 25 & 0,22 & $\begin{array}{c}\text { La incidencia es baja respecto a la población. Se supone } \\
\text { que la } \\
\text { pedanía más cercana al centro está a } 1,5 \mathrm{~km}\end{array}$ \\
\hline $\begin{array}{l}\text { Del desbroce y limpieza } \\
\text { sobre la erosión }\end{array}$ & 43 & 0,62 & La incidencia sólo será sobre la zona de ocupación \\
\hline $\begin{array}{l}\text { De la recogida de RSA } \\
\text { sobre la dinámica de cauces }\end{array}$ & 41 & 0,58 & $\begin{array}{c}\text { Incidencia importante y positiva. Evitamos la } \\
\text { contaminación } \\
\text { de la reserva marina y los posibles cambios de avenidas }\end{array}$ \\
\hline $\begin{array}{l}\text { Del levantamiento de } \\
\text { polvo sobre la deposición }\end{array}$ & 38 & 0,51 & Es un efecto reversible con el asfaltado de los caminos \\
\hline $\begin{array}{l}\text { De las aguas de los envases } \\
\text { de fitosanitarios sobre la } \\
\text { edafología }\end{array}$ & 37 & 0,49 & Se evitará la contaminación del suelo y acuíferos \\
\hline $\begin{array}{l}\text { De la mano de obra } \\
\text { sobre la población }\end{array}$ & 38 & 0,51 & $\begin{array}{l}\text { Incidencia pequeña ya que aunque se genere empleo, } \\
\text { éste será poco relevante pues casi existe pleno empleo }\end{array}$ \\
\hline $\begin{array}{l}\text { De la recogida de RSA sobre } \\
\text { el medio marino y costero }\end{array}$ & 27 & 0,27 & Se evitará la contaminación de la reserva marina \\
\hline $\begin{array}{l}\text { De la recogida de RSA } \\
\text { sobre la población }\end{array}$ & 29 & 0,31 & Incidencia importante pues aumentaría la salubridad \\
\hline $\begin{array}{l}\text { De la recogida de RSA } \\
\text { sobre las áreas de mercado }\end{array}$ & 26 & 0,24 & $\begin{array}{c}\text { Aumentarían posiblemente los precios de los productos } \\
\text { agrícolas de la zona }\end{array}$ \\
\hline
\end{tabular}

8. Funciones de transformación. De izquierda a derecha: a) De localización sobre la población, b) Del desbroce y limpieza sobre la erosión, c) De la recogida de RSA sobre la dinámica de cauces, d) Del levantamiento de polvo sobre la deposición, e) De las aguas de los envases de fitosanitarios sobre la edafología y f) De la mano de obra sobre la población (Gómez, 1999) (17).

\subsection{Medidas correctoras}

Las medidas correctoras (Tabla 10, pág. 89) se realizan sobre los dos impactos moderados y no compatibles (Tabla 8), para lo cual, en la fase de construcción, deberá de ejecutarse el asfaltado de caminos, como consecuencia de la gravedad de la deposición de polvo sobre las cubiertas de invernaderos y cultivos, con la pérdida de producción que eso conlleva por disminución de la eficiencia fotosintética.

Igualmente se deberá impermeabilizar la zona de acopio de envases fitosanitarios con una solera de hormigón polímero, al igual que las conducciones subterráneas y el tanque de actividad que recogerá los vertidos. 
Tabla 8

Valor final del impacto en unidades homogéneas

\begin{tabular}{|c|c|c|c|c|c|}
\hline IMPACTO & $\begin{array}{l}\text { MAG }_{\text {IMP }} \\
\text { Tabla } 5\end{array}$ & $\begin{array}{l}\text { INC }_{\text {EST }} \\
\text { Tabla } 7\end{array}$ & $\begin{array}{l}\text { VALOR } \\
\text { FINAL }\end{array}$ & CALIFICACIÓN & COMENTARIO \\
\hline $\begin{array}{l}\text { De localización } \\
\text { sobre la población }\end{array}$ & $-0,21$ & 0,22 & $-0,05$ & Compatible & \\
\hline $\begin{array}{l}\text { Del desbroce y limpieza } \\
\text { sobre la erosión }\end{array}$ & $-0,23$ & 0,62 & $-0,14$ & Compatible & \\
\hline $\begin{array}{l}\text { De la recogida de RSA sobre } \\
\text { la dinámica de cauces }\end{array}$ & 0,96 & 0,58 & $+0,56$ & Positivo & \\
\hline $\begin{array}{l}\text { Del levantamiento de } \\
\text { polvo sobre la deposición }\end{array}$ & $-0,38$ & 0,51 & $-0,19$ & Moderado & $\begin{array}{l}\text { Se diseñará una batería de medidas correctoras con el fin de } \\
\text { reducir las emisiones de polvo a la atmósfera como } \\
\text { consecuencia del paso de los vehículos por los caminos, tanto } \\
\text { interiores como de acceso a las instalaciones y su posterior } \\
\text { deposición. }\end{array}$ \\
\hline $\begin{array}{l}\text { De las aguas de los } \\
\text { envases de fitosanitarios } \\
\text { sobre la edafología }\end{array}$ & $-0,34$ & 0,49 & $-0,17$ & Moderado & $\begin{array}{l}\text { Se diseñará una batería de medidas correctoras con el fin de } \\
\text { evitar cualquier vertido del contenido de los envases de } \\
\text { productos fitosanitarios al suelo, lo que supondría tanto la } \\
\text { contaminación del mismo, como la posibilidad de afectar al } \\
\text { acuífero subterráneo. Asimismo se evitará la entrada de agua de } \\
\text { lluvia a la nave de almacenaje de envases de productos } \\
\text { fitosanitarios. }\end{array}$ \\
\hline $\begin{array}{l}\text { De la mano de obra } \\
\text { sobre la población }\end{array}$ & 0,03 & 0,51 & $+0,02$ & Positivo & \\
\hline $\begin{array}{l}\text { De la recogida de RSA sobre } \\
\text { el medio marítimo y costero }\end{array}$ & 0,54 & 0,27 & $+0,15$ & Positivo & \\
\hline $\begin{array}{l}\text { De la recogida de RSA sobre } \\
\text { la Población }\end{array}$ & 0,66 & 0,31 & $+0,20$ & Positivo & \\
\hline $\begin{array}{l}\text { De la recogida de RSA sobre } \\
\text { las áreas de mercado }\end{array}$ & 0,44 & 0,24 & $+0,11$ & Positivo & \\
\hline
\end{tabular}

Tabla 9

Impacto final del proyecto

\begin{tabular}{|c|c|c|c|c|}
\hline Factor & $\begin{array}{c}\text { Peso } \\
\text { relativo }\left(\mathbf{P}_{\mathrm{i}}\right)\end{array}$ & Impacto & $\begin{array}{c}\text { Valor del } \\
\text { Impacto }\left(\mathrm{V}_{\mathrm{i}}\right)\end{array}$ & $\begin{array}{c}\text { Valor Ponderado } \\
\left(P_{i} \cdot V_{i}\right) / 1000\end{array}$ \\
\hline Población & 60 & De localización sobre la población & $-0,05$ & $-0,003$ \\
\hline Erosión & 75 & Del desbroce y limpieza sobre la erosión & $-0,14$ & $-0,011$ \\
\hline Dinámica de cauces & 230 & De la recogida de RSA sobre la dinámica de cauces & $+0,56$ & $+0,129$ \\
\hline Deposición & 65 & Del levantamiento de polvo sobre la deposición & $-0,19$ & $-0,012$ \\
\hline $\begin{array}{l}\text { Edafología } \\
\text { del entorno }\end{array}$ & 120 & $\begin{array}{l}\text { De las aguas de los envases de fitosanitarios sobre la } \\
\text { edafología }\end{array}$ & $-0,17$ & $-0,020$ \\
\hline Población & 60 & De la mano de obra sobre la población & $+0,02$ & $+0,001$ \\
\hline $\begin{array}{l}\text { Medio marino } \\
\text { y costero }\end{array}$ & 210 & De la recogida de RSA sobre el medio marino y costero & $+0,15$ & $+0,032$ \\
\hline Población & 60 & De la recogida de RSA sobre la población & $+0,20$ & $+0,012$ \\
\hline Áreas de mercado & 120 & De la recogida de RSA sobre las áreas de mercado & $+0,11$ & $+0,013$ \\
\hline
\end{tabular}

El hormigón polímero es cinco veces más resistente que el hormigón tradicional de cemento, el porcentaje de absorción de agua es nulo frente al 16\% del hormigón tradicional, es resistente a cualquier producto químico y mantiene intactas sus propiedades físicas a pesar de los agentes climatológicos (35).

Con esta última medida se garantiza la no percolación de efluentes de productos químicos al subsuelo y se permite el control y posterior tratamiento de dichos efluentes tal y como describen López y Rodríguez (2003) (9).

Por otro lado, podría ser considerada como medida correctora excepcional la opción de no realizar un centro de transformación y gestión de residuos y destinar todos los residuos sólidos agrícolas a la obtención de energía, como sugiere Elias (2007) (13) y Demirbas, 2005) (14), mediante biogás y/u otro sistema, pero esto requeriría una elevada inversión, además de un coste ecológico adicional (emisiones de dióxido de carbono a la atmósfera y residuos de cenizas).

\subsection{Programa de vigilancia ambiental}

El Plan de Seguimiento y Control tiene como finalidad comprobar la severidad y distribución de los impactos negativos previstos, y especialmente de los no previstos cuando ocurran, para asegurar así el desarrollo de nuevas medidas correctoras, o las debidas compensaciones cuando se necesiten. Al objeto de contrastar y seguir la evolución de lo expuesto se proponen los siguientes puntos relativos a los impactos negativos calificados como moderados o severos.

\subsubsection{Asfaltado de los caminos, tanto interiores como de acceso a las instalaciones y estado de conservación de los mismos}

a) Objetivo 1: evitar el levantamiento de polvo y la posterior deposición del mismo.

- Indicador de realización: \% de la superficie total dedicada a caminos, tanto interiores como de acceso a la finca, que se encuentra asfaltada.

-Frecuencia: controles periódicos en fase de construcción. Periodicidad mínima bimensual para los caminos interiores de la finca, men- 
Tabla 10

Medidas correctoras de los impactos moderados y no compatibles

\begin{tabular}{|c|c|}
\hline IMPACTO & Del levantamiento de polvo sobre la deposición \\
\hline Medida asociada & Asfaltado de los caminos tanto interiores como de acceso a la finca \\
\hline Objetivo & Evitar el levantamiento de polvo y la posterior deposición del mismo \\
\hline Tipo de medida & Obligatoria \\
\hline Carácter & Correctora \\
\hline Fase en que se ejecuta la medida & Fase de construcción \\
\hline $\begin{array}{l}N^{\circ} \text { de impactos o factores a que se } \\
\text { dirige }\end{array}$ & Polivalente (erosión, deposición) \\
\hline Entidad responsable de la medida & $\begin{array}{c}\text { El promotor en el caso de los caminos interiores y la autoridad } \\
\text { competente en el caso de los caminos exteriores. }\end{array}$ \\
\hline Impacto residual & No estimado \\
\hline Impacto de la propia medida & Emisión de ruidos durante los trabajos de asfaltado de los caminos. \\
\hline Coste de inversión & Alto \\
\hline Precauciones de conservación & Asegurar el buen drenaje de los caminos \\
\hline Mantenimiento y control & Control de malas hierbas en las cunetas \\
\hline Urgencia & Alta \\
\hline Documento en que debe incluirse & Proyecto Técnico \\
\hline Facilidad de ejecución & Media \\
\hline Eficacia & Alta \\
\hline IMPACTO & De las aguas de los envases de fitosanitarios sobre la edafología \\
\hline Medida asociada & $\begin{array}{l}\text { Impermeabilización del área donde se almacenarán los envases de fitosanitarios, } \\
\text { recogiendo todos los vertidos en un tanque de actividad para su posterior recogida y } \\
\text { traslado a gestor autorizado para su destoxificación }\end{array}$ \\
\hline Objetivo & $\begin{array}{c}\text { Evitar cualquier percolación de productos que pudieran ocasionar la contaminación del } \\
\text { acuífero y el suelo }\end{array}$ \\
\hline Tipo de medida & Obligatoria \\
\hline Carácter & Correctora \\
\hline Fase en que se ejecuta la medida & Fase de construcción \\
\hline $\begin{array}{l}N^{\circ} \text { de impactos o factores } \\
\text { a que se dirige }\end{array}$ & Polivalente (calidad del suelo, calidad de las aguas subterráneas) \\
\hline Entidad responsable de la medida & Promotor \\
\hline Impacto residual & No estimado \\
\hline Impacto de la propia medida & Pérdida de la estructura del suelo en la zona que quedará impermeable \\
\hline Coste de inversión & Medio 1 r c \\
\hline Precauciones de conservación & $\begin{array}{c}\text { Revisiones periódicas de la estanqueidad del área de almacenamiento de envases de } \\
\text { fitosanitarios }\end{array}$ \\
\hline Mantenimiento y control & $\begin{array}{c}\text { Mantenimiento de conducciones y tanque de actividad. Control del nivel de vertidos en el } \\
\text { tanque de actividad, para evitar que éste pueda rebosar }\end{array}$ \\
\hline Urgencia & Alta \\
\hline $\begin{array}{l}\text { Documento en que } \\
\text { debe incluirse }\end{array}$ & Proyecto Técnico \\
\hline Facilidad de ejecución & Media \\
\hline Eficacia & Muy alta \\
\hline
\end{tabular}

sual en el caso de los caminos exteriores. Los controles continuarán durante la fase de explotación, con periodicidad bimensual.

- Valor Umbral: $\leq 90 \%$ de la superficie total dedicada a los caminos interiores sin asfaltar y $\leq 95 \%$ de la superficie total dedicada a los caminos de acceso a la finca sin asfaltar.

-Momento/os de análisis del Valor Umbral: Presencia ostensible de polvo perceptible por simple observación visual, según criterio del Director Ambiental de la Obra. Se procederá a realizar una inspección adicional, en caso de producirse lluvias torrenciales.

- Medida/as complementarias: hasta que concluyan los trabajos de asfaltado de los caminos, se regarán los mismos diariamente y, si así lo estimara conveniente el Director Ambiental de la Obra, se recubrirán de yeso antes de efectuar el riego sobre ellos.

b) Objetivo 2: reducir el riesgo de accidentes en los caminos tanto interiores como de acceso a la finca.

- Indicador de realización: \% de la superficie total dedicada a caminos, tanto interiores como de acceso a la finca, que se encuentra en perfecto estado de conservación.

- Frecuencia: periodicidad mínima bimensual para los caminos interiores de la finca, mensual en el caso de los caminos exteriores.
- Valor Umbral: $\leq 90 \%$ de la superficie total dedicada a los caminos interiores en perfecto estado de conservación y $\leq 95 \%$ de la superficie total, en el caso de los caminos de acceso a la finca.

- Momento/os de análisis del Valor Umbral: tras cada control.

- Medida/as complementarias: hasta que concluyan los trabajos de asfaltado de los caminos, se limitará la velocidad de circulación en los mismos y se señalizarán convenientemente los tramos de los mismos que se encuentren en obras.

\subsubsection{Protección de la calidad del suelo. Estanqueidad de la nave de almacenamiento de envases de productos fitosanitarios}

a) Objetivo 1: evitar cualquier percolación en la zona de almacenamiento de envases de productos fitosanitarios que pudiera ocasionar la contaminación del suelo.

- Indicador de realización: observación de algún desperfecto que pudiera comprometer la estanqueidad de la zona de almacenamiento de envases de productos fitosanitarios.

-Frecuencia: inspecciones de visu cada vez que se retiren los envases. 
- Valor Umbral: la observación de cualquier desperfecto que pudiera comprometer la estanqueidad de la nave de almacenamiento de envases de productos fitosanitarios se considerará inadmisible.

- Medida/as complementarias: al ser catalogados como residuo muy peligroso (tóxico), se extremarán las medidas de protección personal en el manejo de dichos residuos. - Información a proporcionar por parte del contratista: se requerirá del constructor, la garantía, tanto de los materiales utilizados como del montaje de los mismos.

b) Objetivo 2: Evitar la entrada de agua de lluvia en la nave de almacenamiento de envases de productos fitosanitarios que pudiera ocasionar el rebosamiento del tanque de actividad, lo que provocaría la contaminación del suelo en dicha zona.

- Indicador de realización: observación de algún desperfecto tanto en la cubierta de la nave, como en la red de bajantes encargada de evacuar el agua de lluvia de la cubierta, que pudiera suponer la entrada de agua en la nave.

- Frecuencia: inspecciones de visu cada dos meses, tanto de la cubierta como de los bajantes. Adicionalmente se realizará una nueva inspección un mes antes de la llegada de la época de mayor probabilidad de lluvias.

- Valor Umbral: cualquier desperfecto que pudiera comprometer la estanqueidad, tanto de la cubierta como de los bajantes que evacuarán el agua de lluvia se considerará inadmisible.

- Medida/as complementarias: el sistema de bajantes se construirá con doble tubo, con el fin de obtener mayor seguridad, al reducir el riesgo de roturas.

- Información a proporcionar por parte del contratista: se requerirá del constructor, la garantía, tanto de los materiales utilizados como del montaje de los mismos.

\subsection{Propuesta de medidas correctoras y el programa de vigilancia tanto en la fase de ejecución de la actividad proyectada como en la de funcionamiento}

Desde el punto de vista medioambiental, los principales impactos que implica el proyecto objeto de esta Evaluación de Impacto Ambiental se pueden resumir en los siguientes:

\subsubsection{Impactos negativos}

- Impacto de la localización y emplazamiento sobre la población. Tras su valoración, este impacto obtuvo una calificación de compatible por lo que se considera poco importante; si bien, dada la importancia de la concienciación social en lo referente a temas de conservación del medio ambien- te, lo cual, en último término, es lo que ha dado lugar al desarrollo del Plan de Higiene Rural de la provincia, en donde se engloban las actuaciones objeto del proyecto al cual se refiere la presente Evaluación de Impacto Ambiental, se considera conveniente la adopción de algunas medidas, como pueden ser: mantener reuniones periódicas con los diferentes colectivos y sectores sociales de la provincia con el fin de informar y crear conciencia acerca de la necesidad de llevar a cabo dicho Plan de Higiene Rural; así como de las repercusiones, sin duda positivas, que el desarrollo de dicho Plan conllevará para el conjunto de los diferentes colectivos y actividades presentes en la provincia en los que se refiere a la mejora de la Calidad Medioambiental, la cual revierte en una mejora de la Calidad de Vida del conjunto de los integrantes de la provincia.

- Impacto del desbroce y limpieza del terreno sobre la erosión. Siendo negativo el signo de este impacto, su calificación es de compatible, por lo que en principio es asumible desde el punto de vista medioambiental, si bien en el proyecto objeto de esta Evaluación de Impacto Ambiental estará previsto el ajardinamiento de la zona afectada por el desbroce que resulta imprescindible realizar en la parcela donde se ubicará el Centro de Transformación y Gestión de Residuos Sólidos Agrícolas. Dicho ajardinamiento se contemplará ya en el proyecto como una acción más del mismo, si bien está orientado específicamente a compensar los efectos negativos del impacto que se trata en este apartado.

- Impacto del levantamiento de polvo sobre la deposición. Se trata a continuación uno de los principales impactos identificados en el proyecto, el cual obtuvo la calificación de moderado y para el cual se ha diseñado una batería de medidas correctoras con el fin de reducir las emisiones de polvo a la atmósfera como consecuencia del paso de los vehículos por los caminos, tanto interiores como de acceso a la parcela; así como su posterior deposición. Dichas medidas se concretaron en:

- Asfaltado de todos los caminos, tanto interiores como de acceso a la finca donde se ubicará el Centro de Transformación y Gestión de Residuos Sólidos Agrícolas.

- Riego diario de los caminos, tanto interiores como de acceso al Centro, en tanto en cuanto no se concluyan los trabajos de asfaltado.

- Recubrimiento con yeso de la superficie sin asfaltar de los caminos, previo al riego, en caso de que el Director Ambiental de la Obra lo estime oportuno, hasta que concluyan los trabajos de asfaltado.

- Impacto de las aguas de restos de productos fitosanitarios en envases sobre la edafología del entorno. Nos referimos en este 
apartado al Impacto más negativo de todos cuantos se han identificado en esta Evaluación de Impacto Ambiental y que ha obtenido una calificación de moderado. Por este motivo se han diseñado una serie de medidas correctoras encaminadas a evitar por un lado cualquier vertido del contenido de los envases de productos fitosanitarios al suelo, lo que supondría tanto la contaminación del mismo, como la posibilidad de afectar al acuífero subterráneo.

De entre estas medidas destacan:

- Impermeabilización del área donde se almacenarán los envases de fitosanitarios.

- Recogida de cualquier vertido en un tanque de actividad para su posterior recogida y traslado a gestor autorizado que proceda a su destoxificación.

- Puesta a punto y exigencia del cumplimiento de las medidas de protección personal en el manejo de los envases de productos fitosanitarios.

- Evitar la entrada de agua de lluvia en la nave de almacenamiento de envases de productos fitosanitarios que pudiera ocasionar el rebosamiento del tanque de actividad.

- Revisión periódica del nivel del tanque de actividad.

- Revisión periódica de la estanqueidad de la nave destinada al almacenamiento de envases de productos fitosanitarios.

- El sistema de bajantes se construirá con doble tubo, con el fin de obtener mayor seguridad, al reducir el riesgo de roturas.

\subsubsection{Impactos positivos}

- Impacto de la recogida de Residuos Sólidos Agrícolas sobre la dinámica de cauces. La importancia de este impacto, calificado como severo, radica, por una parte, en la extensión de la zona que se verá afectada por el mismo, la cual comprende todos los cauces existentes en la provincia; $y$, por otra ,en el estado que ellos pueden presentar debido a que los agricultores de no existir el centro de residuos depositarán en los cauces los mismos.

- Impacto de la recogida de Residuos Sólidos Agrícolas sobre el medio marino y costero. Al igual que en el caso anterior, nos encontramos aquí frente a un impacto de gran importancia ya que afecta de forma directa a la franja litoral de la provincia que, en gran parte, se encuentra catalogada como Parque Natural y/o Reserva Marina, de aquí que se le haya dado un gran peso relativo frente al resto de los impactos causados por el proyecto. A este respecto, la Recogida de RSA supondrá que se dejen de verter al medio marino y costero una ingente cantidad de residuos.

- Impacto de la recogida de Residuos Sólidos Agrícolas sobre la población. No por obvia debemos dejar de señalar la gran trascendencia de este impacto, que actúa de forma directa sobre la Calidad de Vida de los habitantes de la provincia, en que:

- Evitará la quema indiscriminada e incontrolada de los RSA, lo que repercutirá en una mayor calidad del aire en la zona. Del mismo modo se evitará la generación de malos olores como consecuencia de los restos de productos y material vegetal en proceso de descomposición.

- El control de envases fitosanitarios y su gestión eliminará los riesgos que se pudiesen producir de un modo eventual sobre la contaminación de las aguas.

- Evitará el actual contacto entre la población y un gran número de residuos de diversas características, entre los que se encuentran algunos considerados como muy tóxicos, con el riesgo que ello supone.

- Permitirá la futura recuperación de gran cantidad de zonas que debido al estado que presentan actualmente no son utilizables por la población.

- Se prevé que la retirada de RSA provoque el descenso en la densidad de plagas agrícolas que actualmente se registran en la provincia y que resulta un grave problema para el desarrollo de la actividad agrícola. Este descenso en la densidad de plagas agrícolas provocará, a su vez, un descenso en la presión de tratamientos fitosanitarios, lo que al mismo tiempo supondrá la disminución del tiempo de exposición personal de los trabajadores agrícolas a los productos que se utilizan para este fin.

- Desde un punto de vista higiénico-sanitario, se eliminará el riesgo de que los menores puedan utilizar envases de productos fitosanitarios para sus juegos poniendo en peligro su salud.

- Asimismo la retirada de RSA supondrá un descenso en la densidad de roedores, moscas y mosquitos los cuales, además de un problema para la actividad agrícola, supone un riesgo para la salud pública, al ser éstos, vectores de enfermedades que llegan a afectar al hombre.

- En otro orden de cosas, los RSA que actualmente se encuentran diseminados por toda la provincia y que en muchos casos son tóxicos, sirven actualmente como alimento al ganado, caprino y ovino, de algunas explotaciones de la zona, con el consiguiente riesgo para la salud pública que esto supone. Se prevé que la retirada de RSA termine con esta peligrosa situación.

- La gestión de RSA tiene un efecto inmediato sobre el paisaje ya que se elimina el abandono incontrolado de los mismos en cualquier lugar. 
- Impacto de la recogida de Residuos Sólidos Agrícolas sobre las áreas de mercado. Como consecuencia del rumbo que están tomando los mercados de frutas y hortalizas, en la actualidad verdadero motor de la economía de la provincia, en el sentido de demandar productos libres de residuos y obtenidos a través de prácticas respetuosas con el medio ambiente, rechazando o penalizando vía precios aquéllos que no se producen en las condiciones adecuadas, resulta obvia la importancia de la recogida de RSA de las explotaciones agrícolas como un primer paso para lograr una producción que se adecue a las exigencias de mercado, así como para conseguir la sostenibilidad del modelo agrícola implantado.

- Impacto de la contratación de mano de obra en el Centro de Transformación y Gestión de Residuos Sólidos Agrícolas. El centro de transformación y gestión de residuos sólidos agrícolas deberá situarse en zonas de baja densidad de población, así la contratación de personal supondrá una mejora en la renta de la población que habita esta área. En este sentido se procurará la contratación del personal necesario para el desarrollo de la actividad, entre dicha población, siempre que el nivel de cualificación requerido lo permita.

\section{CONCLUSIONES}

La obtención de energía a partir de residuos sólidos agrícolas contamina menos que otro tipo de procesos, pero desde el punto de vista medioambiental es más ventajoso compostar, reciclar y reutilizar.

No obstante, estas acciones llevan consigo unas reacciones que pueden llegar a ser molestas para la población y profesionales de la agricultura, tales como: levantamiento de polvo y su posterior deposición, lixiviados, malos olores y una localización de la actividad no deseada, entre otras.

Las medidas correctoras propuestas en este estudio: impermeabilización del lugar de acopio de los envases de productos fitosanitarios con hormigón polímero garantizando la estanqueidad de dicha zona, asfaltado de caminos, divulgación y concienciación de la población y ajardinamientos, entre otras, repercutirán en la mejor ejecución y desarrollo de la actividad, favoreciendo la limpieza de los cauces, la menor contaminación del medio marino y rural, la mayor calidad paisajística, la venta de productos hortícolas más respetuosos con el medio (en toda la provincia) y la contratación de mano de obra.

\section{REFERENCIAS}

(1) Sanjuán, J. F., 2004.: Estudio Multitemporal sobre la Evolución de la Superficie Invernada en la Provincia de Almería por Términos Municipales desde 1984 hasta 2004. Ed. Isabel María Cuadrado Gómez de FIAPA, Almería, 97 pp.

(2) Hall, D. O., House, J. 1995.: Biomass - an environmentally acceptable fuel for the future. Proceedings of the Institution of Mechanical Engineers Part A-. Journal of Power and Energy. Vol. 209 (3): 203-213.

(3) Velázquez-Martí, B. Aprovechamiento de los residuos forestales para uso energético. Ed. UPV. ISBN: 97-8848-363049-5. Ed. UPV. ref. 2006.799.

(4) Velázquez-Martí, B., Fernández-González, E. 2009. Analysis of the process of biomass harvesting with collecting-chippers fed by pick up headers in plantations of olive trees. Biosystems engineering 103 (4): 184-190.

(5) Camacho Ferre, F., Callejón Ferre, A. J., Fernández Rodríguez, E., Montoya García, M. E., Moreno Cascó, J., Valverde García, A., Galán López, M. y Rodríguez Rodríguez, M. P. 2000.: Estudio Técnico de Plan de Higiene Rural. Término Municipal de Níjar. Ed. Mónsul Ingeniería S.L., Almería, España. 554 pp.

(6) Lightfoot, C., Noble, R. 2001.: Tracking the ecological soundness of farming systems: Instruments and indicators. Journal of Sustainable Agriculture. Vol. 19 (1): 9-29.

(7) Velázquez-Martí, B. y Annevelink, E. 2009. GIS application to define biomasws collection points as sources for linear programming of delivery networks. Transactions of ASABE 52 (4): 1069-1078.

(8) Velázquez-Martí, B. y Fernández-González, E. 2010. Mathematical algorithms to locate factories to transform biomass in bioenergy focused on logistic network construction. Renewable Energy 35: 21362142.

(9) López, J. A., Rodríguez, A. 2003.: Prototipo de planta de tratamiento integral para el reciclado y valoración de los residuos de envases de productos fitosanitarios. VI Congreso Ibérico-Americano de Engenharia Mecánica-Cibem6. Ed. A. M. Dias. Coimbra. Portugal.

(10) Matarán, A. 2006.: Análisis de los Impactos ambientales producidos por los invernaderos. Residuos. Vol. 94, 2-13.

(11) Antón, A., Castells, F., Montero, J. I. 2007.: Land use indicators in life cycle assessment. Case study: The environmental impact of Mediterranean greenhouses. Journal of Cleaner Production. Vol. 15 (5): 432-438.

(12) Ros, M., García, C., Hernández, M.T. 2007.: Evaluation of different pig slurry composts as fertilizer of horticultural crops: Effects on selected chemical and microbial properties. Renewable Agriculture and Food Systems. Vol. 22 (4): p. 307-315. 
(13) Elias, X. 2007.: Is considered the biomass as an energetic alternative?. DYNA . Vol. 82 (1): 41-43.

(14) Demirbas, A. 2005.: Potential applications of renewable energy sources, biomass combustion problems in boiler power systems and combustion related environmental issues. Progress in Energy and Combustion Science. Vol. 31 (2) p. 171-192.

(15) Velázquez-Martí, B., Fernández-González, E., Estornell, J., Ruiz, L.A. 2010. Dendrometric and dasometric analysis of the bushy biomass in Mediterranean forests. Forest Ecology and Management 259: 875-882.

(16) Gómez, D. 1988.: Evaluación del Impacto Ambiental de Proyectos Agrarios. Ed. Instituto de Reforma y Desarrollo Agrario (IRYDA). Madrid. 270 pp. ISBN: 84-7479-677-6

(17) Gómez, D. 1999.: Evaluación de Impacto Ambiental. Ed. Mundiprensa y Editorial Agrícola Española. Madrid. $701 \mathrm{pp}$.

(18) Girardin, P., Bockstaller, C., Van Der Werf, H. 2000.: Assessment of potential impacts of agricultural practices on the environment: the AGRO*ECO method. Environmental impact. Assessment Review 20, 227-239.

(19) Braband, D., Geier, U., Kopke, U. 2003.: Bio-resource evaluation within agri-environmental assessment tools in different European countries. Agriculture Ecosystems \& Environment. Vol. 98 (1-3): 423-434.

(20) Krishnan, N., Raoux, S., Dornfeld, D. 2004.: Quantifying the environmental footprint of semiconductor equipment using the environmental value systems analysis (EnV-S). IEEE Transactions on Semiconductor Manufacturing. Vol. 17 (4): 554-561.

(21) Payraudeau, S., Van der Werf, H. M. G. 2005.: Environmental impact assessment for a farming region: a review of methods. Agriculture Ecosystems \& Environment. Vol. 107 (1): 1-19.

(22) Van der Werf, H. M. G., Tzilivakis, J., Lewis, K., Basset-Mens, C. 2007.: Environmental impacts of farm scenarios according to five assessment methods. Agriculture Ecosystems \& Environment. Vol. 118 (1-4): 327-338.

(23) Galán, M. B., Peschard, D., Boizard, H.: 2007. ISO 14001 at the farm level: Analysis of five methods for evaluating the environmental impact of agricultural practices. Journal of Environmental Management. Vol. 82 (3): 341-352.

(24) Anderson, M.J., Thompson, A. A. 2007.: Multivariate control charts for ecological and environmental monitoring. Ecological Applications. Vol. 14 (6): 1921-1935.

(25) Williams, C. L., Hargrove, W. W., Liebman, M., James, D. E. 2008.: Agro-ecoregionalization of lowa using multivariate geographical clustering. Agriculture Ecosystems \& Environment. Vol. 123 (1-3): 161-174.

(26) De León Llamazares, A. 1986.: Atlas Agroclimático Nacional de España; Tomos I, Il y III. Ed. MAPA. Madrid.

(27) BOE (2008) Real Decreto Legislativo 1/2008, de 11 de enero, por el que se aprueba el texto refundido de la Ley de Evaluación de Impacto Ambiental de proyectos. Boletín Oficial del Estado No. 23, 26/01/2008.

(28) BOJA, 2007. Ley 7/2007, de 9 de julio, de gestión integrada de la calidad ambiental de Andalucía. Boletín Oficial de la Junta de Andalucía No. 143, 20/07/2007.

(29) Callejón-Ferre, A.J., Pérez-Alonso, J., Sánchez-Hermosilla, J., Carreño-Ortega, A. 2009a. Ergonomics and psycho-sociological quality indeces in greenhouses, Almería (Spain). Spanish Journal of Agricultural Research, Vol. 7 (1): 50-58.

(30) Fernández, C. y Pérez, J. J. 2004.: Caracterización de los invernaderos de la provincial de Almería. Ed. Cajamar. Almería, 20 pp.

(31) Fundación Cajamar. 2006. Análisis de la campaña hortofrutícola de Almería 2005/2006. Cajamar (Caja Rural Intermediterránea), Almería, España. 38 pp.

(32) Callejón-Ferre, A.J., Manzano Agugliaro, F., Díaz-Pérez, M., Carreño-Ortega, A., Pérez-Alonso, J. 2009b. Effect of shading with aluminised screens on fruit production and quality in tomato (Solanum lycopersicum L.) under greenhouse conditions. Spanish Journal of Agricultural Research, Vol. 7 (1): 41-49.

(33) Manzano, F. 2007. Gasificación de residuos de invernadero para la obtención de energía eléctrica en el sur de España: Ubicación mediante SIG. Interciencia. Vol. 32 (2): 131-136.

(34) Callejón-Ferre, A.J. and López-Martínez, J.A. 2009. Briquettes of plant remains from the greenhouse of Almería (Spain). Spanish Journal of Agricultural Research, Vol. 7 (3): 525-534.

(35) http://www.polimerosherna.com/ficha_tecnica.pdf. Disponible el 21/10/2008. 